\title{
Recurrent Interstitial pregnancy: a new approach in diagnosis with MRI and management with systemic methotrexate along with intra foetal cardiac $\mathbf{1 0} \%$ potassium chloride
}

\author{
Prasad Lele $^{1 *}$, Manoj Kumar Tangri ${ }^{2}$, Deepak Patil $^{3}$, Rajesh Sharma ${ }^{3}$
}

\begin{abstract}
${ }^{1}$ Department of Obstetrics and Gynaecology, Command Hospital, King Gorge Medical University, Lucknow, India ${ }^{2}$ Department of Obstetrics and Gynaecology, Command Hospital, West Bengal Medical University, Kolkata, India ${ }^{3}$ Department of Obstetrics and Gynaecology, Command Hospital, Pune, India
\end{abstract}

Received: 18 November 2015

Accepted: 07 January 2016

\section{*Correspondence:}

Dr. Prasad Lele,

E-mail: prasadlele@ hotmail.com

Copyright: ( ) the author(s), publisher and licensee Medip Academy. This is an open-access article distributed under the terms of the Creative Commons Attribution Non-Commercial License, which permits unrestricted non-commercial use, distribution, and reproduction in any medium, provided the original work is properly cited.

\begin{abstract}
We present a case of recurrent interstitial pregnancy in a setting of recurrent pregnancy loss where we used a new modality in the diagnosis and treatment. The patient after diagnosis of interstitial pregnancy by Ultrasonography was very keen to continue her pregnancy so the diagnosis was confirmed with MRI imaging showing the "interstitial sign" that convinced her for termination. In this case presence of active foetal cardiac activity and high Serum $\beta$ hCG (43000 $\mathrm{mIU} / \mathrm{ml}$ ), made medical management a difficult proposition. Then we conceived a hybrid combined medical management of intra-foetal cardiac $10 \% \mathrm{KCl}$ and adjuvant systemic multi-dose MTX therapy. This resulted in resolution of ectopic and conserves her fertility.
\end{abstract}

Keywords: Interstitial pregnancy, Interstitial sign, MRI, Hybrid combined systemic and foetal intra cardiac approach

\section{INTRODUCTION}

Interstitial pregnancy is ectopic implantation of the gestation sac in the uterine cornu or interstitial portion of fallopian tube. This great masquerader is found in $2 \%$ to $4 \%$ of ectopic pregnancies. ${ }^{1}$ The interstitial portion of the fallopian tube is embedded in the uterine musculature and is a very vascular area. The area being juxtaposition to the endometrial cavity, implantation of gestational sac here is generally diagnosed late in the pregnancy. Because of the rarity, if the diagnosis is missed then the pregnancy grows into the second trimester and may result in catastrophic uterine rupture thus compromising patients fertility.

Ultrasonography is an important tool for diagnosis of interstitial pregnancy but may miss done cautiously. ${ }^{2}$ Here we present a case of recurrent pregnancy loss who was very keen on having a baby presenting with recurrent interstitial ectopic. We used a new approach to the investigation (MRI) and conservative hybrid medical management in presence of foetal cardiac activity and raised serum $\beta$ hCG levels.

\section{CASE REPORT}

A 27 year old lady (Gravida 5 Para 0) presented at 08 weeks 04 days period of amenorrhea with complaints of spotting per vagina. She had three spontaneous first trimester abortions in the past. In her last pregnancy, she had left interstitial ectopic pregnancy conceived after IUI, and managed with multidose methotrexate treatment.

This was her fifth pregnancy, a spontaneous conception while under treatment for recurrent pregnancy loss. Pregnancy was diagnosed at home with a urine pregnancy test. On imaging with ultrasonography, we found a single gestation sac eccentrically placed towards the right cornu 
of the uterus with CRL corresponding to 8 weeks and presence of foetal cardiac activity suggesting right interstitial pregnancy and the patient was advised termination of pregnancy.

The lady was very keen to continue the pregnancy and so to confirm the diagnosis of interstitial pregnancy MRI was done. The MRI showed a foetus in the interstitial portion of the fallopian tube and a thin line "interstitial line" separating the endometrial cavity from the fetus. (Figure $1 \& 2$ ) Termination of pregnancy was advised to the patient but in view of high serum $\beta$ hCG (43000IU) and presence of foetal cardiac activity medical management was difficult.

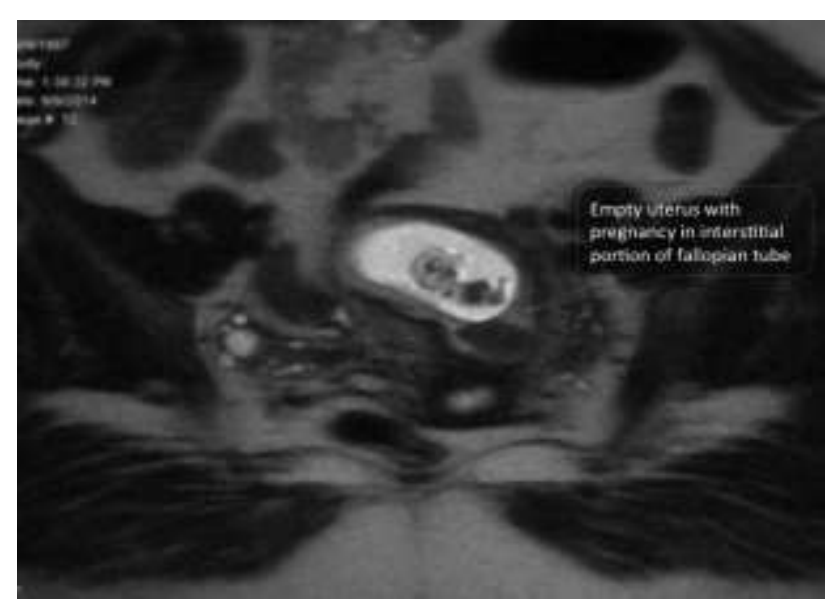

Figure 1: Showing empty uterus and pregnancy in interstitial portion of fallopian tube.

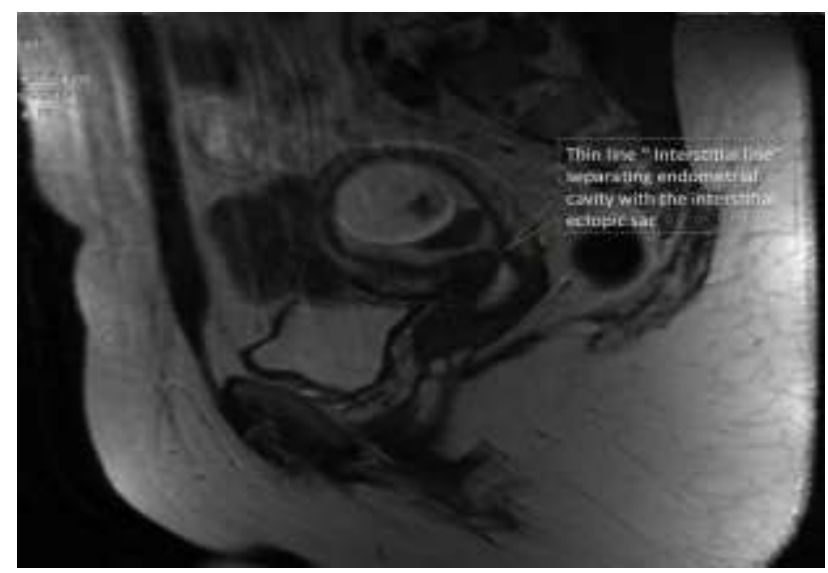

Figure 2: "Interstitial line" a thin line seperating endometrial cavity from the interstitial ectopic sac.

Surgical management of this case was not the correct preferred modality of treatment as she had previous four pregnancy losses and fertility preservation was her prime concern. The presence of foetal cardiac activity and high $\beta \mathrm{hCG}$ did not favour for systemic multidose methotrexate therapy alone and a hybrid combined approach with foetal intra cardiac injection of $10 \%$ Potassium Chloride was considered.
After her consent patient was taken up for intra foetal cardiac injection of $10 \% \mathrm{KCL}$ under sonographic guidance in the operation theatre. The patient was positioned in the lithotomy position under total intravenous anaesthesia. Through Trans vaginal approach under ultrasonographic guidance with help of embryo reduction needle the foetal heart was approached and $0.3 \mathrm{ml}$ of $10 \% \mathrm{KCL}$ was injected. The foetal cardiac asystole was confirmed by power Doppler and re checked after 24 hours.

In view of high serum $\beta$ hCG (43000IU) above treatment was considered insufficient and was followed by adjuvant systemic multidose methotrexate with folinic acid. The patient was given Inj Methotextrate $50 \mathrm{mg}$ intramuscular on Day 1, 3, 5 and 7 with folinic acid rescue of $15 \mathrm{mg}$ intramuscular on Day 2, 4, 6 and 8. Her serum $\beta$ hCG levels on the fifth and seventh day of treatment came down to 23641 and 10064 respectively. The ultrasonographic evaluation showed creneated resolving ectopic gestation sac. The Patient remained comfortable and was discharged on the $11^{\text {th }}$ day with serum $\beta \mathrm{hCG}$ levels 2500. On follow up OPD visits the patient was asymptomatic and had complete resolution of the ectopic gestational sac.

\section{DISCUSSION}

Interstitial pregnancy is indeed a rare ectopic pregnancy with diagnosed on the basis of clinical history, Serum $\beta$ hCG levels and USG. But the diagnosis is often delayed and pregnancy may reach an advanced gestation because of the interstitial uterine muscular wall allowing it to grow up to 14 to 16 weeks of gestation. The rupture of such an advanced gestation may result in fatal catastrophic haemorrhage in $2 \%$ cases. $^{3}$

Ultrasonography is remains the principal modality for diagnosis of interstitial pregnancy. ${ }^{4}$ Timor-Tritsch et al. (1992) for the sonographic diagnosis of interstitial pregnancy suggested to see an "empty uterus with a gestational sac that is seen separately and $<1 \mathrm{~cm}$ from the most lateral edge of the uterine cavity and a thin myometrial layer surrounding the sac". Tanaka Y et al (2014) emphasized the importance of three-dimensional ultrasonography (3D TVS), ${ }^{6}$ with its capacity to reproduce the coronal plane of the uterus and assist the exact localization of the gestational sac relative to the uterine cornu. The eccentric location of the gestational sac and superior and lateral myometrial stripes is better and easily visualized on coronal scans generated through 3D TVS.

When TVS findings are indeterminate, magnetic resonance imaging (MRI) may further improve delineation of the focus of ectopic owing to its excellent tissue contrast. $^{7}$ Key MRI features of interstitial pregnancy include an eccentric cystic structure which represents a gestational sac with foetal pole "a classically hypointense cystic appearance T1, T2 hyperintensity 
surrounded a ring" in the interstitial portion of fallopian tube is away from the sagittal axis of the uterus as we saw in our case (Figure 1). There is a myometrial coat around the gestational sac and thin hypointense line separates the gestation sac from the endometrium on $\mathrm{T} 2 \mathrm{~W}$ images this represents the 'interstitial line' sign seen on ultrasound images (Figure 2).

Surgical management may not be the preferred modality of treatment in the cases where fertility preservation is the issue as in our case. Anatomical abnormalities in like fibroids in the cornual region of the uterus, may make the management even more difficult. Laparotomy or laparoscopic approaches with or without robotic assistance can be used for cornual resection. ${ }^{8}$

Practice Committee of the American Society for Reproductive Medicine (2013) came with a committee opinion that MTX can be used to treat ectopic pregnancies that occur in cervical, cornual and caesarean scar locations, and it does not adversely affect ovarian reserve or subsequent fertility. ${ }^{9}$

Presence of fetal cardiac activity may be present despite decreasing titer of $\beta$ HCG after MTX therapy necessitating the surgical management. ${ }^{10}$ Also there have been published reports of rupture ectopic following systemic MTX therapy in interstitial ectopic requiring emergency surgical management. ${ }^{11}$ Swank ML et al described successful management of three cases of interstitial ectopic pregnancies with combined, intraamniotic or intra fetal $\mathrm{KCl}$ and systemic approach using methotrexate to prevent surgery ${ }^{12}$ whereas in our case we had done foetal intracardiac injection of $10 \% \mathrm{KCl}$.

In our case presence of foetal cardiac activity, high serum $\beta$ hCG levels > $43000 \mathrm{mIU} / \mathrm{ml}$ were predictors for failure of MTX therapy. These parameters prompted us to conceive a hybrid combined approach of TVS guided foetal intra cardiac injection of $10 \% \mathrm{KCL}$ and systemic adjuvant MTX multidose therapy.

There are very few cases reported in literature where MRI has been used in diagnosis of Interstitial Pregnancy when TVS picture is indeterminate. Also we could not find any literature where local foetal intracardiac $\mathrm{KCl}$ injection (which is superior to intrafetal or intra-amniotic injection) combined local and systemic approach presented was used.

Patients presenting with advanced live ectopic gestation interstitial pregnancy MRI can adjunct diagnosis. Ibid combined medical management can be used in clinically stable patients and surgical intervention may be avoided.
Funding: No funding sources

Conflict of interest: None declared

Ethical approval: Not required

\section{REFERENCES}

1. Valsky DV, Yagel S. Ectopic pregnancies of unusual location: management dilemmas. Ultrasound Obstet Gynecol. 2008;31(3):245-51.

2. Bourdel N, Roman H, Gallot D, Lenglet Y. Interstitial Pregnancy. Ultrasonographic diagnosis and contribution of MRI. A case report. Gynecol Obstet Fertil. 2007;35(2):121-4.

3. Faraj R, Steel M. Review Management of cornual (interstitial) pregnancy. Obstet \& Gynecol. 2007;9:249-55.

4. Lin EP, Bhatt S, Dogra VS. Diagnostic clues to ectopic pregnancy. Radiographics. 2008;28(6): 166171.

5. Timor-Tritsch IE, Monteagudo A, Matera C, Veit CR. Sonographic evolution of cornual pregnancies treated without surgery. Obstet Gynecol. 1992;79:1044-9.

6. Tanaka Y, Mimura K, Kanagawa T, Kajimoto E, Takahashi K, Kakigano A. Three-Dimensional Sonography in the Differential Diagnosis of Interstitial, Angular, and Intrauterine Pregnancies in a Septate Uterus. JUM. 2014;33:2031-5.

7. Tamai K, Koyama T, Togashi K. MR features of ectopic pregnancy. Eur Radiol. 2007;17(12):323646.

8. Ansari A, Ahmad S, James JA, Jeppson CN, Holloway RW. Robotic-assisted laparoscopic resection of cornual ectopic pregnancy. A case report. J Reprod Med. 2015;60(1-2):58-64.

9. Pfeifer S, Goldberg J, Lobo R, Thomas M, Widra E,Licht M. Medical treatment of ectopic pregnancy: a committee opinion. Fertil Steril. 2013;100(3):63844.

10. Szylit NA, Podgaec S, Traina E, Oliveira Rde C. Video laparoscopic intervention for an interstitial pregnancy after failure of clinical treatment. Sao Paulo Med J. 2012;130(3):202-7.

11. Naredi N, Sandeep K, Jamwal VDS. Interstitial implantation in a setting of recurrent ectopic pregnancy: A rare combination. MJAFI. 2015;71:194-6.

12. Swank ML, Harken TR, Porto M Management of interstitial ectopic pregnancies with a combined intra-amniotic and systemic approach. Obstet Gynecol. 2013;122(2):461-4.

Cite this article as: Lele $\mathrm{P}$, Tangri MK, Patil D, Sharma R. Recurrent Interstitial pregnancy: a new approach in diagnosis with MRI and management with systemic methotrexate along with intra foetal cardiac $10 \%$ potassium chloride. Int J Reprod Contracept Obstet Gynecol 2016;5:539-41. 